\title{
THE ALGEBRA GENERATED BY NILPOTENT ELEMENTS IN A MATRIX CENTRALIZER*
}

\author{
RALPH JOHN DE LA CRUZ ${ }^{\dagger}$ AND ELOISE MISA $\dagger$
}

\begin{abstract}
For an arbitrary square matrix $S$, denote by $C(S)$ the centralizer of $S$, and by $C(S)_{N}$ the set of all nilpotent elements in $C(S)$. In this paper, we use the Weyr canonical form to study the subalgebra of $C(S)$ generated by $C(S)_{N}$. We determine conditions on $S$ such that $C(S)_{N}$ is a subalgebra of $C(S)$. We also determine conditions on $S$ such that the subalgebra generated by $C(S)_{N}$ is $C(S)$.
\end{abstract}

Key words. Nilpotent, Weyr form, Centralizer, Matrix decompositions.

AMS subject classifications. 15A21, 15A23, 15A27.

1. Introduction. An element $N$ of a ring $R$ is nilpotent if $N^{k}=0$ for some positive integer $k$. Several papers have been devoted to determining when an element of a ring or algebra is a sum or product of its nilpotent elements. In [6], it was shown that every commutator in a simple ring with a nontrivial idempotent is the sum of nilpotent elements of index 2 . It was also shown that this does not always hold true for simple rings with nontrivial zero-divisors or prime rings with nontrivial idempotents, which answers a question of Herstein in [8]. Expressing commutators as sums of nilpotent elements were also considered for a unital algebra generated by its idempotents and for a von Neumann algebra in [1], for simple Artinian rings in [7], and for unital $\mathrm{C}^{*}$-algebra with nontrivial projections satisfying certain conditions in [11]. In the algebra $\mathcal{B}(\mathcal{H})$ of operators on a complex, separable, infinite-dimensional Hilbert space $\mathcal{H}$, products of two nilpotent operators were considered in [9]. It was shown that an operator $T$ is a product of two nilpotent operators if and only if $\operatorname{dim} \operatorname{ker}(T)=\operatorname{dim} \operatorname{ker}\left(T^{*}\right)=\infty$. The case when these nilpotent operators commute and are both square-zero was studied in [5]. It was shown that $T$ is a product of two commuting square-zero operators if and only if $T$ is a square-zero operator and the $\operatorname{dim}\left(\operatorname{ker}(T) \cap \operatorname{ker}\left(T^{*}\right)\right)=\infty$.

In this work, we consider matrix rings and decompositions of square matrices into sums and products of nilpotent matrices. It was shown in [7] that there exist division rings $D$ such that every $n \times n$ matrix $A$ over $D$ is a sum of nilpotent matrices for $n \geq 2$. In [15], Wang and Wu showed that a square matrix $A$ over a complex Hilbert space is a sum of two nilpotent matrices of index 2 if and and only if $A$ is similar to $-A$. This was generalized to an arbitrary field by Botha in [2]. In [3], Breaz and Călugăreanu showed that if $A$ is an $n \times n$ traceless matrix over a commutative $\operatorname{ring} R$, then $A$ is a sum of three nilpotent matrices. Moreover, when $R$ is replaced by a field $F$, it was shown that if $\operatorname{char}(F)=0$ or $\operatorname{char}(F)$ does not divide $n$, then $A$ is a sum of two nilpotent matrices. And, if $\operatorname{char}(F)$ divides $n$, then $A$ is not a sum of two nilpotent matrices if and only if $A=\lambda I$ for some $\lambda \neq 0$. In addition, it was shown in [13] that every traceless square matrix over an arbitrary field $\mathrm{F}$ is a sum of four nilpotent matrices of index 2 . If $\operatorname{char}(\mathrm{F})=2$, the number of summands for such a decomposition is three. In [16], Wu showed that any complex singular square matrix

* Received by the editors on July 26, 2021. Accepted for publication on December 5, 2021. Handling Editor: João Filipe Queiró. Corresponding Author: Ralph John de la Cruz.

$\dagger$ Institute of Mathematics, University of the Philippines, Diliman, Quezon City, Philippines (rjdelacruz@math.upd.edu.ph, misa.eloise@gmail.com). 
$A$ is a product of two nilpotent matrices with ranks both equal to rank $A$, except when $A$ is a $2 \times 2$ nilpotent matrix of rank 1 . This was shown to be true for an arbitrary field in [14]. In [12], Novak characterized the matrices over an algebraically closed field $F$ that are products of two or three nilpotent matrices of index 2 . It was shown that $A \in M_{n}(F)$ is a product of three nilpotent matrices of index 2 if and only if rank $A \leq \frac{n}{2}$. In [10], Laffey considered the case of integer matrices and proved that for $n \geq 3$, any singular $n \times n$ matrix $A$ over $\mathbb{Z}$ is a product of nilpotent integer matrices.

For an arbitrary square matrix $S$, denote by $C(S)$ the centralizer of $S$, that is, the algebra of all matrices $A$ such that $A S=S A$, and by $C(S)_{N}$ the set of all nilpotent elements in $C(S)$. In this paper, we study the subalgebra of $C(S)$ generated by $C(S)_{N}$. We determine conditions on $S$ such that $C(S)_{N}$ is a subalgebra of $C(S)$ (see Corollary 3.6). We also determine conditions on $S$ such that the subalgebra generated by $C(S)_{N}$ is $C(S)$ (see Corollary 3.5). We make use of the Weyr canonical form to prove our results. There is an overarching theorem from the structure of finite-dimensional algebras $R$ over a field $F$ from which most of the results follow very quickly when expressed in terms of the Weyr form. This is called the Wedderburn's Principal Theorem established in 1908, which says that when $F$ is perfect, such $R$ decomposes as a semidirect product of its radical $\operatorname{rad}(R)$ and its semisimple part $S$. However, the structure of $R / \operatorname{rad}(R)(\cong S)$ was first established in 1961 by Gerstenhaber using the Jordan form in [4]. We present this decomposition using the Weyr form in Theorem 3.1. In Section 2, we discuss the Weyr canonical form, and we review basic properties of $C(S)$ when $S$ is in Weyr canonical form. In Section 3, we prove our main results.

2. Preliminaries. Throughout this paper, we let $F$ be an arbitrary field. Denote by $M_{n}(F)$ the set of all $n \times n$ matrices over $F$.

Definition 2.1 ([17, Definition 2.1.1]). A basic Weyr matrix with eigenvalue $\lambda$ is an $n \times n$ matrix $W$ of the following form: There is a partition $n_{1}+\cdots+n_{r}=n$ of $n$ with $n_{1} \geq \cdots \geq n_{r} \geq 1$ such that, when $W$ is viewed as an $r \times r$ blocked matrix $\left[W_{i j}\right]$, where the $(i, j)$ block $W_{i j}$ is an $n_{i} \times n_{j}$ matrix, the following three features are present:

1. The main diagonal blocks $W_{i i}$ are the scalar matrices $\lambda I_{n_{i}}$ for $i=1, \ldots, r$.

2. The first superdiagonal blocks $W_{i, i+1}$ are full column-rank $n_{i} \times n_{i+1}$ matrices in reduced row-echelon form (i.e., an identity matrix $I_{n_{i+1}}$ followed by zero rows) for $i=1, \ldots, r-1$.

3. All the other blocks of $W$ are zero (i.e., $W_{i j}=0$ when $\left.j \neq i, i+1\right)$.

In this case, we say that $W$ has Weyr structure $\left(n_{1}, \ldots, n_{r}\right)$. The Weyr structure is homogeneous if $n_{1}=$ $n_{2}=\cdots=n_{r}$. Note that if $W$ is a homogeneous basic Weyr matrix with eigenvalue $\lambda$, then $r=1$ if and only if $W=\lambda I$. For example, the following is a $7 \times 7$ basic Weyr matrix with eigenvalue $\lambda$ and Weyr structure $(3,2,1,1)$ :

$$
W=\left[\begin{array}{ccc|cc|c|c}
\lambda & 0 & 0 & 1 & 0 & 0 & 0 \\
0 & \lambda & 0 & 0 & 1 & 0 & 0 \\
0 & 0 & \lambda & 0 & 0 & 0 & 0 \\
\hline & & & \lambda & 0 & 1 & 0 \\
& & & 0 & \lambda & 0 & 0 \\
\hline & & & & \lambda & 1 \\
\hline & & & & & \lambda
\end{array}\right] .
$$


Definition 2.2. A square matrix $W$ is a Weyr matrix, or is in Weyr form, if $W$ is a direct sum of basic Weyr matrices with distinct eigenvalues. That is, if $\lambda_{1}, \ldots, \lambda_{k}$ are the distinct eigenvalues of $W$, then $W$ has the form:

$$
W=\left[\begin{array}{llll}
W_{1} & & & \\
& W_{2} & & \\
& & \ddots & \\
& & & W_{k}
\end{array}\right],
$$

where $W_{i}$ is a basic Weyr matrix with eigenvalue $\lambda_{i}$ for $i=1, \ldots, k$.

Note that, by definition, a Weyr matrix with a single eigenvalue is a basic Weyr matrix.

TheOREM 2.3 ([17, Theorem 2.2.4]). To within permutations of basic Weyr blocks, each square matrix $S$ over an algebraically closed field is similar to a unique Weyr matrix $W$. The matrix $W$ is called the Weyr canonical form of $S$.

Let $S \in M_{n}(F)$ and $W$ be the Weyr canonical form of $S$, then $W=X S X^{-1}$ for some nonsingular matrix $X$. Note that $A S=S A$ if and only if $\left(X A X^{-1}\right) W=X A S X^{-1}=X S A X^{-1}=W\left(X A X^{-1}\right)$, which implies

$$
C(W)=X C(S) X^{-1}=\left\{X A X^{-1} \mid A \in C(S)\right\}
$$

Hence, there is a one-to-one correspondence between the elements of $C(S)$ and $C(W)$. Note that a matrix $A \in C(S)$ is a sum or product of matrices in $C(S)_{N}$ if and only if $X A X^{-1}$ is a sum or product of matrices in $C(W)_{N}$. Indeed, let $A \in C(S)$ and suppose $A=N_{1}+\cdots+N_{m}$ or $A=N_{1} N_{2} \cdots N_{m}$ for some $N_{1}, \ldots, N_{m} \in$ $C(S)_{N}$. Then,

$$
X A X^{-1}=X\left(N_{1}+\cdots+N_{m}\right) X^{-1}=X N_{1} X^{-1}+\cdots+X N_{m} X^{-1},
$$

or

$$
X A X^{-1}=X\left(N_{1} N_{2} \cdots N_{m}\right) X^{-1}=\left(X N_{1} X^{-1}\right)\left(X N_{2} X^{-1}\right) X \cdots X^{-1}\left(X N_{m} X^{-1}\right),
$$

where $X N_{i} X^{-1} \in C(W)_{N}$ for all $i=1, \ldots, m$. That is, $X A X^{-1} \in C(W)$ is a sum or product of matrices in $C(W)_{N}$. The converse is shown similarly. Thus, there is also a one-to-one correspondence between the elements of the subalgebra $\mathcal{A}(S)$ of $C(S)$ generated by $C(S)_{N}$ and the subalgebra $\mathcal{A}(W)$ of $C(W)$ generated by $C(W)_{N}$. And so, we assume without loss of generality that $S$ is in Weyr form. Let $\lambda_{1}, \ldots, \lambda_{k}$ be the distinct eigenvalues of $S$, and say

$$
S=S_{1} \oplus \cdots \oplus S_{k}
$$

where $S_{i}$ is a basic Weyr matrix with eigenvalue $\lambda_{i}$ for $i=1, \ldots, k$. Suppose $A$ commutes with $S$ and partition $A=\left[A_{i j}\right]_{1 \leq i, j \leq k}$ conformal to $S$. Since $A S=S A$, we have $A_{i j} S_{j}=S_{i} A_{i, j}$ for all $i$ and $j$. Note that when $i=j$, we have $A_{i i} S_{i}=S_{i} A_{i i}$, and so for each $i=1, \ldots, k, A_{i i}$ commutes with $S_{i}$. Now, suppose that $i \neq j$. Since $\lambda_{i}$ is the only eigenvalue of $S_{i}$, it follows from the Cayley-Hamilton theorem that $\left(S_{i}-\lambda_{i} I\right)^{m}=0$ for some $m \in \mathbb{N}$. Then, we have

$$
A_{i j}\left(S_{j}-\lambda_{i} I\right)^{m}=\left(S_{i}-\lambda_{i} I\right)^{m} A_{i j}=0
$$

Since $\lambda_{i}$ is not an eigenvalue of $S_{j}$, then $S_{j}-\lambda_{i} I$ is nonsingular. It follows from (2.2) that $A_{i j}=0$ for $i \neq j$. Hence, if $A$ commutes with $S$, then

$$
A=A_{11} \oplus \cdots \oplus A_{k k}
$$


where $A$ is partitioned conformal to $S$ and each $A_{i i} \in C\left(S_{i}\right)$. We also have that $A$ is a sum or product of elements in $C(S)_{N}$ if and only if each $A_{i i}$ is a sum or product of elements in $C\left(S_{i}\right)_{N}$. Thus, we further assume without loss of generality, that $S$ is a basic Weyr matrix. The following gives a description of the elements of a centralizer of a basic Weyr matrix.

Lemma 2.4 ([17, Proposition 2.3.3]). Let $W$ be an $n \times n$ basic Weyr matrix with Weyr structure $\left(n_{1}, \ldots, n_{r}\right), r \geq 2$. Let $K=\left[K_{i j}\right]$ be an $n \times n$ matrix partitioned conformal to $W$. Then $W$ and $K$ commute if and only if $K$ is a block upper triangular matrix for which

$$
K_{i j}=\left[\begin{array}{cc}
K_{i+1, j+1} & * \\
0_{n_{i}-n_{i+1}, n_{j+1}} & *
\end{array}\right] \text { for } 1 \leq i \leq j \leq r-1 .
$$

Here, we have written $K_{i j}$ as a blocked matrix where the zero block is $\left(n_{i}-n_{i+1}\right) \times n_{j+1}$. (The column of asterisks is absent if $n_{j}=n_{j+1}$, and the $[0 *]$ row is absent if $n_{i}=n_{i+1}$.)

Hence, $K \in C(W)$ is nilpotent if and only if $K_{i i}$ is nilpotent for all $i$.

3. Main results. A wonderful thing about the special block upper triangular form of a basic Weyr matrix $W$ is that it gives us a natural way of decomposing any matrix that centralizes $W$ into a sum of a nilpotent and a diagonal matrix. The conclusions of Wedderburn's Principal Theorem, mentioned in the Introduction, still hold in the case of $\mathrm{R}=\mathrm{C}(\mathrm{W})$ without any restriction on the field $\mathrm{F}$, due to the explicit upper triangular form of the centralizer of a nilpotent Weyr matrix W. Consider the $9 \times 9$ nilpotent Weyr matrix $W$ with Weyr structure $(4,2,2,1)$ so that

$$
W=\left[\begin{array}{llll|ll|ll|l}
0 & 0 & 0 & 0 & 1 & 0 & 0 & 0 & 0 \\
0 & 0 & 0 & 0 & 0 & 1 & 0 & 0 & 0 \\
0 & 0 & 0 & 0 & 0 & 0 & 0 & 0 & 0 \\
0 & 0 & 0 & 0 & 0 & 0 & 0 & 0 & 0 \\
\hline & & & & 0 & 0 & 1 & 0 & 0 \\
& & 0 & 0 & 0 & 1 & 0 \\
\hline & & & 0 & 0 & 1 \\
& & & & & & 0 & 0 \\
\cline { 3 - 6 } & & & & & & & &
\end{array}\right],
$$

and centralizing matrices of $W$ take the form:

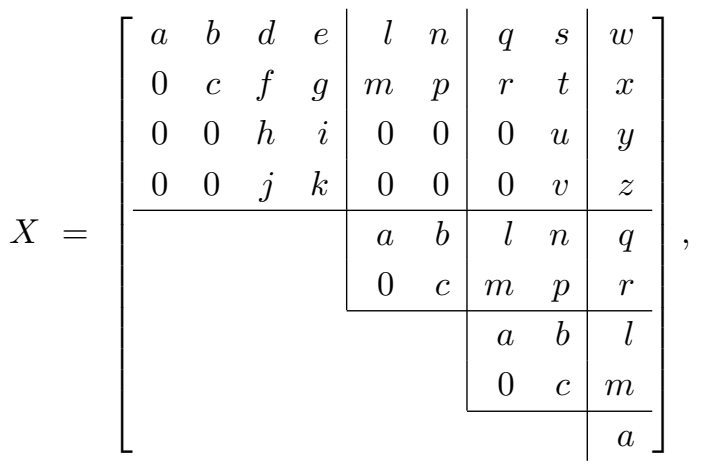


for arbitrary $a, b, c, \ldots, z \in F$. Then

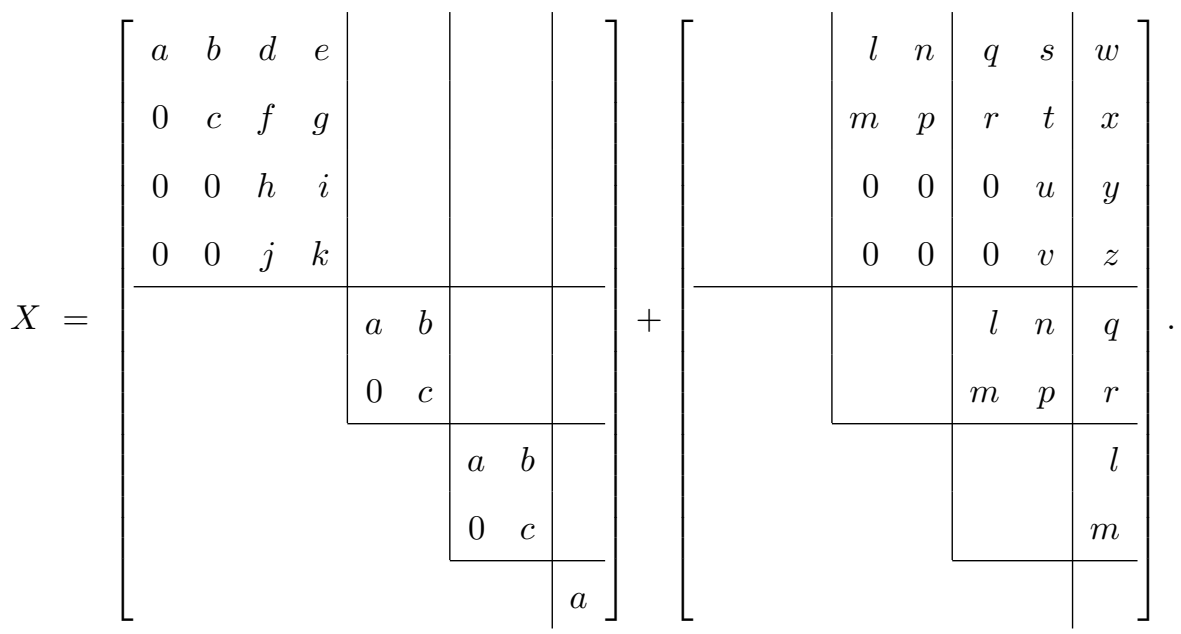

The above gives us a decomposition of $C(W)$ as $T+S$, where $S$ consists of block diagonal matrices as in (3.6) and $T$ consists of nilpotent block matrices of the form as in (3.6). The following theorem, from which all of the important results of this paper follow, tells us that we can further refine the partition of the structure of $W$ as in (3.5) so that $T$ has optimal dimension.

Theorem 3.1. Let $W$ be an $n \times n$ nilpotent Weyr matrix over a field $F$, and let $R=\mathcal{C}(W)$ be the algebra of $n \times n$ matrices centralizing $W$. Let $x=\left(n_{1}, n_{2}, \ldots, n_{r}\right)$ be the Weyr structure of $W$, and let $k_{1}=n_{1}, k_{2}, \ldots, k_{s}=n_{r}$ be the distinct $n_{i} s$ from largest to smallest. For convenience, set $k_{s+1}=0$. Using the special block upper triangular form of $\mathcal{C}(W)$, refine the partition $x$ so that $R$ becomes block upper triangular whose diagonal blocks have the following form:

1. The blocks have size $n_{r} \times n_{r}$ and $\left(n_{j}-n_{j+1}\right) \times\left(n_{j}-n_{j+1}\right)$ for $j=1,2, \ldots, r-1$ if $n_{j}>n_{j+1}$, but with some repetitions.

2. The distinct blocks have size $\left(k_{i}-k_{i+1}\right) \times\left(k_{i}-k_{i+1}\right)$ for $i=1, \ldots, s$.

3. The entries in the distinct blocks are arbitrary.

Let $T$ be the strictly upper block triangular part of $R$ with respect to the refined partition, and let $S$ be the block diagonal part. Then

$$
T=\operatorname{rad}(R) \quad \text { and } \quad R=T \oplus S \quad \text { as } F \text {-spaces, }
$$

so $R$ is the semi-direct product of the ideal $T$ and subalgebra $S$. Consequently,

$$
R / \operatorname{rad}(R) \cong S \cong \prod_{i=1}^{s} M_{k_{i}-k_{i+1}}(F) .
$$

Our description of $R / \operatorname{rad}(R)$ is the dual of that given by Gerstenhaber in terms of the Jordan structure $\left(m_{1}, m_{2}, \ldots, m_{t}\right)$ of $W$ (which is the dual partition of $x$ ). The sizes of the matrix rings involved are given in terms of the number of times each distinct Jordan structure component $m_{j}$ is repeated in the structure.

Proof. The refined partition is described on [18, p. 208]. They call it the standard partition. We illustrate with an example. Consider the matrix $W$ from (3.5) with structure $x=(4,2,2,1)$. Now blocking the typical centralizing matrix relative to the refined partition $(1,1,2,1,1,1,1,1)$ yields the desired form of 


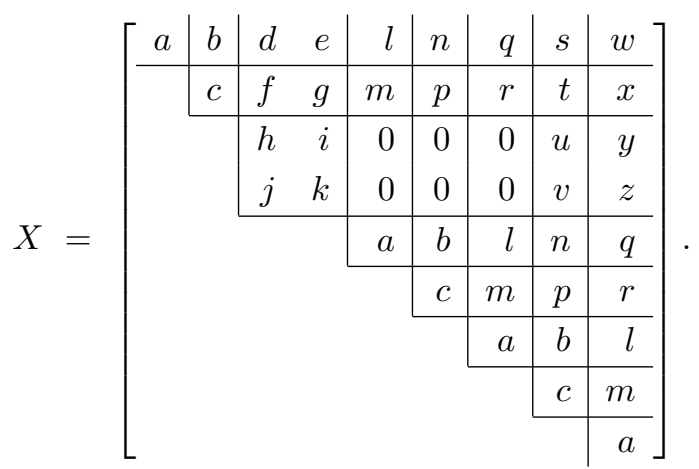

(Note that with the standard partition, the diagonal blocks are no longer in decreasing order of size.) The distinct diagonal blocks are

$$
[a],[c],\left[\begin{array}{cc}
h & i \\
j & k
\end{array}\right]
$$

whence

$$
R / \operatorname{rad}(R) \cong F \times F \times M_{2}(F)
$$

Returning to the general proof, it is clear that $T$ is a nilpotent ideal of $R, R=T \oplus S$, and $S \cong$ $\prod_{i=1}^{s} M_{k_{i}-k_{i+1}}(F)$. Since $S$ is semisimple, it has no nonzero nilpotent ideals. Therefore, $T$ must be the maximum nilpotent ideal of $R$ thereby making $T=\operatorname{rad}(R)$.

Corollary 3.2. Let $A$ be the subalgebra of $R$ generated by its nilpotent elements. Then

$$
\begin{aligned}
& n_{1}^{2}+n_{2}^{2}+\cdots+n_{r}^{2}-\left(k_{1}-k_{2}\right)^{2}-\left(k_{2}-k_{3}\right)^{2}-\cdots-\left(k_{s-1}-k_{s}\right)^{2}-k_{s}^{2} \\
& \leq \operatorname{dim} A \\
& \leq n_{1}^{2}+n_{2}^{2}+\cdots+n_{r}^{2}
\end{aligned}
$$

and these bounds are tight.

Proof. Clearly, $\operatorname{rad}(R) \subseteq A \subseteq R$, so $\operatorname{dim} \operatorname{rad}(R) \leq \operatorname{dim} A \leq \operatorname{dim} R$. However, $\operatorname{dim} R=n_{1}^{2}+n_{2}^{2}+\cdots+n_{r}^{2}$, as demonstrated in [17, Proposition 3.2.2]. And the claimed lower bound is the dimension of $\operatorname{rad}(R) \operatorname{from}$ Theorem 3.1 .

COROLlaRY 3.3. Any property involving sums or products of nilpotent elements of $R$ (such as the number required for a general member) immediately reduces to the full matrix rings $M_{k_{i}-k_{i+1}}(F)$.

Proof. In the semi-direct product $R=T \oplus S$, each $r \in R$ has a unique expression as $r=t+s$ for $t \in T$ and $s \in S$, and the expression for a product is

$$
r_{1} r_{2}=\left(t_{1}+s_{1}\right)\left(t_{2}+s_{2}\right)=\left(t_{1} t_{2}+t_{1} s_{2}+s_{1} t_{2}\right)+s_{1} s_{2}
$$

because $T$ is an ideal. An immediate consequence is that the product $r_{1} r_{2}$ of nilpotents is nilpotent exactly when $s_{1} s_{2}$ is nilpotent. Likewise for sums.

COROLLARY 3.4. The structure of $R / \operatorname{rad}(R)$ can be an arbitrary finite direct product of full matrix rings over $F$. That is, given a product $M_{m_{1}}(F) \times M_{m_{2}}(F) \times \cdots \times M_{m_{t}}(F)$, there exists a nilpotent matrix $W$ in some $M_{n}(F)$ whose centralizer modulo its radical is isomorphic to the given product. 
Proof. Take the nilpotent Weyr matrix $W$ of structure:

$$
x=\left(m_{1}+m_{2}+\cdots+m_{t}, m_{2}+m_{3}+\cdots+m_{t}, \ldots, m_{t-1}+m_{t}, m_{t}\right) .
$$

and apply Theorem 3.1.

Corollary 3.5. Assume $F$ is not the two element field. Then $R$ is generated by its nilpotent elements if and only if

$$
k_{i} \geq k_{i+1}+2 \text { for } i=1, \ldots, s \text {, }
$$

equivalently,

$$
n_{r} \geq 2, \quad \text { and } n_{j}=n_{j+1} \text { or } n_{j} \geq n_{j+1}+2 \text { for } j=1, \ldots, r-1 .
$$

Proof. Since $F$ is not the two element field, a matrix $\operatorname{ring} M_{m}(F)$ is generated by its nilpotents exactly when $m>1$. The corollary now follows from Theorem 3.1 and Corollary 3.3.

COROLlary 3.6. The subalgebra of $R$ generated by its nilpotent elements equals the set of all nilpotents of $R$ exactly when

$$
k_{i}=k_{i+1}+1 \text { for } i=1, \ldots, s \text {, }
$$

equivalently,

$$
n_{r}=1 \text { and } n_{j} \leq n_{j+1}+1 \text { for } j=1, \ldots, r-1 \text {. }
$$

Proof. Apply Corollary 3.3 and note that in a matrix ring $M_{m}(F)$, all sums and products of nilpotents are nilpotent if and only if $m=1$.

Acknowledgment. The first author is supported by the Crisostomo B. and Cristina C. Garcia Distinguished Centennial Professorial Chair in Mathematics. The second author is supported by DOSTASTHRDP. The authors would like to thank the referee for suggesting the current proof of our main results. The original version was longer and relied heavily on the special form of the elements of the centralizer of a basic Weyr matrix. The current one is shorter and proves a more general result.

\section{REFERENCES}

[1] J. Alaminos, J. Extremera, A.R. Villena, M. Breŝar, and Ŝ. Ŝpenko. Commutators and square-zero elements in Banach algebras. Q. J. Math., 67:1-13, 2016.

[2] J. Botha. Sums of two square-zero matrices over an arbitrary field. Linear Algebra Appl., 436:516-524, 2012.

[3] S. Breaz and G. Clugreanu. Sums of nilpotent matrices. Linear Multilinear Algebra, 65:1-12, 2016.

[4] M. Gerstenhaber. On dominance and varieties of commuting matrices. Ann. Math., 73:324-348, 1961.

[5] D. Kokol Bukovek, T. Koir, N. Novak, and P. Oblak. Products of commuting nilpotent operators. Electron. J. Linear Algebra, 16:237-247, 2007.

[6] M.A. Chebotar, P.-H. Lee, and E. Puczylowski. On commutators and nilpotent elements in simple rings. Bull. Lond. Math. Soc., 42:191-194, 2010.

[7] B. Harris. Commutators in division rings. Proc. Am. Math. Soc., 4:628-630, 1958.

[8] I.N. Herstein. Lie and Jordan structures in simple associative rings. Bull. Amer. Math. Soc., 67:517-531, 1961.

[9] R. Drnovek, V. Mller, and N. Novak. An operator is a product of two quasi-nilpotent operators if and only if it is not semi-Fredholm. Proc. Roy. Soc. Edinburgh Sect. A, 136:935-944, 2006.

[10] T.J. Laffey. Factorizations of integer matrices as products of idempotents and nilpotents. Linear Algebra Appl., 120:81-93, 1989.

[11] L.W. Marcoux. On the linear span of the projections in certain simple C*-algebras. Ind. Univ. Math. J., 51:753-771, 2002.

[12] N. Novak. Products of square-zero operators. J. Math. Anal. Appl., 339:10-17, 2008.

[13] C.D.S. Pazzis. A note on sums of three square-zero matrices. Linear Multilinear Algebra, 65:787-805, 2016.

[14] A.R. Sourour. Nilpotent factorization of matrices. Linear Multilinear Algebra, 31:303-308, 1991. 
[15] J.H. Wang and P.Y. Wu. Sums of square-zero operators. Stud. Math. 99:115-127, 1991.

[16] P.Y. Wu. Products of nilpotent matrices. Linear Algebra Appl. 96:227-232, 1987.

[17] K. O'Meara, J. Clark, and C. Vinsonhaler. Advanced Topics in Linear Algebra: Weaving Matrix Problems Through the Weyr Form. Oxford University Press, Oxford, 2011.

[18] R.A. Horn and C.R. Johnson. Matrix Analysis, Second Edition. Cambridge University Press, New York, 2013.

[19] R.A. Horn and C.R. Johnson. Topics in Matrix Analysis. Cambridge University Press, Cambridge, 1991. 